\title{
A major concern of then: banana Sigatoka disease
}

Among the five articles published in the first two Fruits issues of 1958, three were devoted to banana Sigatoka disease, including two papers in the continuation of a long series on chemical control at a low volume, which has already been treated here. We are reproducing the introductions of these two articles, which give a good idea of the current context:

In Fruits vol. 13 (1), one could read: "Whereas the first chapter of this series, with J. Cuille and H. Guyot, studied the implementation of the spraying material, we approach now the fungicide itself. The fungicidal or fongistatic role of oil has been highlighted since 1955, but some experimenters could wonder what was the advantage of adding a classical fungicide, what was its effectiveness and in which circumstances it was useful to do so.

The work by J. Brun gives the precise details awaited on this point and its rigorous experimentation has a particular value in an area where Sigatoka disease is a major concern.

It would be worth testing the action of mineral oils at a low volume on other crop diseases, because few fungicides are currently sufficiently active to stop an advanced fungic attack, like oil does."

Otherwise, the second article in Fruits vol. 13 (2) specified: "Whereas the second paper of this series was devoted by J. Brun to the fongitoxic action of mineral oils, we are now looking for the determining factor of their plant toxicity."

The work by J. Cuille and B. Blanchet constitutes a preliminary stage aiming at defining the physicochemical properties of oils, particularly important for the induction of plant symptoms. The aim is to look for a wise choice of mineral oils to minimize the effects of true accidents due to an excessive use of these products. Other researchers are looking at the influence of sprays with adequate oil amounts on the plant physiology. Their results will be published later on."

The third article dedicated to banana Sigatoka disease, written by G. Bouriquet and J. Bataille, dealt with two banana parasitic fungi in Madagascar: Cercospora musae and Alternaria musae. Thus, again Sigatoka disease was treated in its conidian form.

Dr. Jacky Ganry

Chairman of the Section on Tropical and Subtropical Fruits

ISHS 


\section{Lu dans Fruits, il y a 50 ans...}

\section{Une préoccupation d'alors : la maladie de Sigatoka du bananier}

Sur les cinq articles publiés dans les deux premiers numéros de Fruits de l'année 1958, trois étaient consacrés à la cercosporiose du bananier, dont deux se situaient dans la suite d'une longue série sur les traitements pesticides à débit réduit dont il a déjà été question ici. Nous reprendrons les deux introductions de chacun de ces deux derniers articles qui résument bien la situation :

Dans Fruits vol. 13 (1), on pouvait lire : "Alors que le premier chapitre de cette série, avec J. Cuillé et H. Guyot, étudiait la mise en ouvre du matériel de traitement, nous abordons maintenant le produit fongicide lui-même. Le rôle fongicide ou fongistatique de l'huile avait été mis en évidence dès 1955, mais des expérimentateurs pouvaient se demander quel intérêt présentait l'addition d'un fongicide classique, quelle était son efficacité et dans quelles circonstances il était nécessaire d'y avoir recours.

L'étude de J. Brun apporte les précisions attendues sur ce point et son expérimentation rigoureuse dans une région où le Cercospora sévit durement possède une valeur particulière.

Il serait intéressant de vérifier l'action des builes minérales utilisées en brouillard léger sur d'autres parasites des plantes cultivées, car peu de fongicides sont actuellement d'une efficacité suffisante pour arrêter une attaque fongique à un stade avancé, comme le fait l'buile."

L'article de Fruits vol. 13 (2) précisait par ailleurs : "Alors que le deuxième mémoire de cette série était consacré par. Brun à l'action fongitoxique des builes minérales, nous abordons maintenant la recherche des facteurs déterminant leur toxicité pour le végétal.

L'étude de J. Cuillé et B. Blanchet constitue une étape préliminaire visant à définir les propriétés physico-chimiques des huiles, particulièrement importantes quant à l'apparition de symptômes graves pour la plante. Il s'agit de tenter de minimiser les répercussions de véritables accidents dus à un emploi exagéré d'builes minérales par un choix judicieux de ces produits. L'influence de l'application de doses normales d'builes sur la physiologie de la plante a été également abordée par d'autres chercheurs qui publieront leurs résultats ultérieurement. "

Le troisième article dédié à la cercosporiose, dû à G. Bouriquet et J. Bataille, traitait de deux champignons parasite du bananier à Madagascar : Cercospora musae et Alternaria musae. La cercosporiose était donc à nouveau traitée dans la forme conidienne de cette importante maladie.

Dr. Jacky Ganry Président de la section Fruits tropicaux et subtropicaux 